\title{
Metazoários Parasitos de Elops saurus (Osteichthyes: Elopiformes: Elopidae) do litoral de Angra dos Reis, Rio de Janeiro, Brasil
}

\author{
Elops saurus (Osteichthyes: Elopiformes: Elopidae) Metazoan Parasites \\ from the coast zone of Angra dos Reis, Rio de Janeiro, Brazil
}

\author{
1 Sara da Silva Coêlho Celestino saracelest@hotmail.com \\ 2 Dimitri Ramos Alves
}

1 Discente do curso de Ciências Biológicas do Centro Universitário de Volta Redonda, UniFOA.
2 Docente do Centro Universitário de Volta Redonda, UniFOA.

\section{Resumo}

Entre agosto de 2014 e janeiro de 2015, foram necropsiados 50 espécimes de Elops saurus (Osteichthyes: Elopiformes: Elopidae), provenientes da Baía da Ponta da Ribeira (230'5.355"S, $\left.44^{\circ} 21^{\prime} 17.628^{\prime \prime} 0\right)$, Angra dos Reis, Estado do Rio de Janeiro, Brasil, sendo necropsiados para estudo de sua comunidade de metazoários parasitos. Três espécies de metazoários parasitos foram coletadas: uma de nematoide e duas de copépodes. Elops saurus é um novo registro de hospedeiro para a larva do nematoide Contracaecum sp. (Anisakidae). Trinta e cinco (70\%) espécimes de $E$. saurus estavam parasitados por, pelo menos, uma espécie de metazoário. Um total de 159 espécimes de parasitos pertencentes a três espécies foi coletado, com abundância média de $3,2 \pm 4,2$. Lernanthropus rathbuni correspondeu à espécie com o maior número de espécimes coletados, sendo a espécie dominante, com maior prevalência, abundância, intensidade e intensidade média. Os copépodes e o nematoide corresponderam a $95,6 \%$ e $4,4 \%$ do total de espécimes de parasitos coletados, respectivamente. Os componentes da comunidade parasitária do $E$. saurus apresentaram o típico padrão de distribuição superdisperso (ID = 5,628; $d=13,631$ ). Não foi observada correlação entre o comprimento total do hospedeiro e a prevalência e abundância parasitárias. A abundância média $\left(r_{s}=0,184 ; P=0,199\right)$ e a riqueza parasitária $\left(r_{s}=0,204 ; P=0,153\right)$ não apresentaram relação com o comprimento total do hospedeiro.

\section{Palavras-chave}

Elopiformes; Elopidae; Elops saurus; Metazoários parasitos.

\begin{abstract}
From August 2014 to January 2015, 50 specimens of ladyfish, Elops saurus (Osteichthyes: Elopiformes: Elopidae), collected from Baía da Ponta da Ribeira

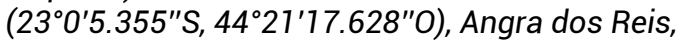
state of Rio de Janeiro, Brazil, were necropsied for the study of their metazoan parasites communities. Three metazoan parasites species were collected: one nematode and two copepods. Elops saurus is a new host record for the nematode Contracaecum sp. (Larva) (Anisakidae). Thirty five (70\%) fishes were parasitized by at least one metazoan species. One hundred fifty-nine specimens, with an average of $3.2 \pm$ 4.2. per host, were collected. Lernanthropus rathbuni constituted the majority of specimens collected and was the dominant species, with highest prevalence, abundance, intensity and mean intensity. The majority of the collected parasites specimens were copepods (95.6\%), followed by the nematode (4.4\%). The parasites species showed typical over-dispersed pattern of distribution (ID $=5.628 ; d=13.631)$. No correlation was observed between the total length of the host and the prevalence and parasitic abundance. The average abundance $\left(r_{s}=0.184, P=0.199\right)$ and the parasite richness $\left(r_{s}=0.204, P=0.153\right)$ were not associated with the total length of the host.
\end{abstract}

\section{Keywords}

Elopiformes; Elopidae; Elops saurus; Metazoan Parasites.

\section{Como você deve citar?}

CELESTINO, Sara da Silva Coêlho; ALVES, Dimitri Ramos. Metazoários Parasitos de Elops saurus (Osteichthyes: Elopiformes: Elopidae) do litoral de Angra dos Reis, Rio de Janeiro, Brasil. Cadernos UniFOA, Volta Redonda, n. 30, p. 109-115, abr. 2016. 


\section{INTRODUÇÃO}

A Ordem Elopiformes é composta de peixes ósseos distribuídos em duas famílias (Elopidae e Megalopidae) e com apenas dois gêneros (Elops e Megalops). Os representantes dessas famílias são tipicamente marinhos, raramente encontrados em ambientes dulcícolas, ocorrendo em mares tropicais e subtropicais (FIGUEIREDO, MENEZES, 1978; WHITEHEAD, VERGARA, 1978; MENEZES et. al., 2003; NELSON, 2006, McBRIDE et al., 2010; ADAMS et. al., 2013).

Elops saurus Linnaeus, 1766 é o único representante da família Elopidae no Brasil. Conhecido como "Ubarana", essa espécie se distribui no Atlântico Ocidental, desde Cape Cod (Massachusetts) até o sudeste brasileiro, sendo mais comum na região norte e nordeste do Brasil. Atinge cerca de 90 $\mathrm{cm}$ de comprimento, habita águas costeiras e alimenta-se de peixes de pequeno porte e crustáceos (FIGUEIREDO, MENEZES, 1978; LOPES, 1989; MENEZES et. al., 2003; ADAMS et. al., 2013).

Embora seja uma espécie muito comercializada no Município de Angra dos Reis, Rio de Janeiro, sua fauna parasitária é pouco conhecida. Os estudos sobre os metazoários parasitos de $E$. saurus foram realizados no Atlântico Norte. Dentre eles, podemos destacar Pearse (1952), com o registro de copépodes; Linton (1890), Palm e Overstreet (2000), com o registro de cestoides e; por Corkum (1959) e Bullard (2014), com digenéticos.

O presente trabalho tem como objetivo desenvolver um estudo da comunidade de metazoários parasitos de Elops saurus proveniente de Angra do Reis, Estado do Rio de Janeiro, realizando uma análise ao nível de infracomunidade e comunidade componente.

\section{MATERIAL E MÉTODOS}

Entre agosto de 2014 e janeiro de 2015, foram necropsiados 50 espécimes de E. saurus (Figura 1), provenientes da Baía da Ponta da Ribeira, sob as coordenadas $23^{\circ} 0^{\prime} 5.355^{\prime \prime} S, 44^{\circ} 21^{\prime} 17.628^{\prime \prime} 0$, Angra dos Reis, Estado do Rio de Janeiro, Brasil. Os espécimes foram coletados por pescadores artesanais e profissionais. Uma vez obtidos, os peixes foram acondicionados em caixas de isopor contendo gelo, para assegurar boas condições da coleta dos parasitos e protegê-los durante o transporte até o Laboratório de Zoologia do Centro Universitário de Volta Redonda (UniFOA), onde foram realizadas as necropsias.

O presente projeto foi submetido e aprovado pelo CEUA/UniFOA ( $N^{\circ} 002 / 14$ - CEUA/UniFOA).

Todos os órgãos e cavidades do corpo foram examinados à procura de parasitos. A coleta, registro e processamento dos helmintos e crustáceos parasitos foram feitas de acordo com os procedimentos indicados por Amato et al. (1991) e Eiras et al. (2000). Os espécimes de hospedeiros foram identificados conforme estudos de Figueiredo e Menezes (1978). 
Figura 1 - Espécime de Elops saurus.

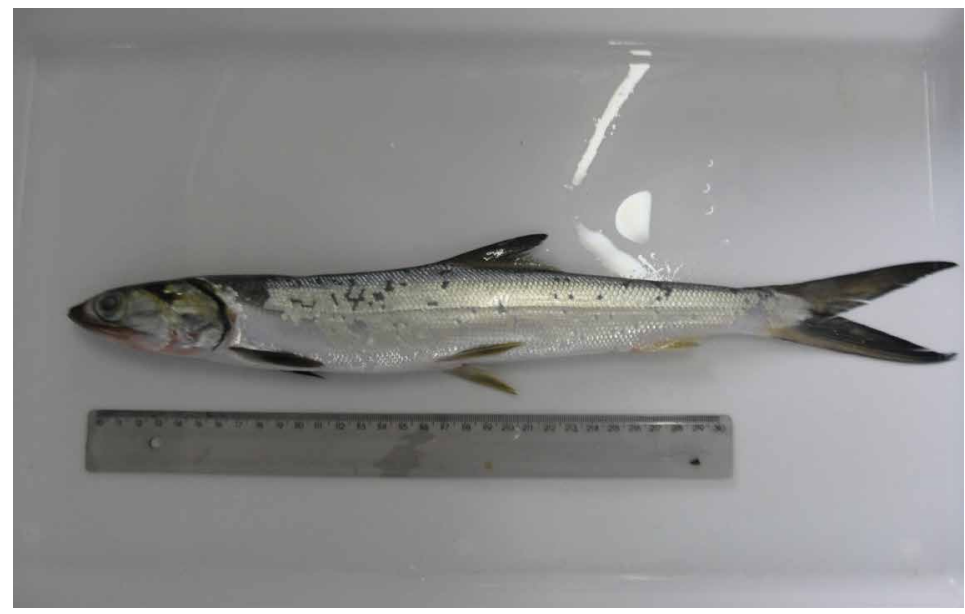

(Foto: Sara da Silva)

A análise estatística foi aplicada somente para as espécies com prevalência maior que 10\% (BUSH et al., 1990). 0 cálculo da frequência de dominância e da dominância relativa (número de espécimes de uma espécie/número total de espécimes de todas as espécies de cada infracomunidade) foi feito seguindo a metodologia de Rohde et al. (1995). O quociente entre a variância e a abundância média (índice de dispersão) foi calculado para cada espécie de parasito, com o intuito de determinar seu padrão de distribuição, sendo sua significância testada com o estatístico d (LUDWIG, REYNOLDS, 1988).

Os dados referentes ao comprimento total dos hospedeiros, número total de parasitos, abundância, riqueza e diversidade parasitária foram transformados logaritmicamente $(\log x+1)$ para aproximação à distribuição normal (ZAR, 1996).

O coeficiente de correlação por postos de Spearman, $r_{s^{\prime}}$ foi usado para determinar possíveis correlações entre o comprimento total do hospedeiro e a abundância de infecção/infestação. 0 coeficiente de correlação de Pearson, $r$, foi usado para determinar a possível correlação entre o comprimento total do hospedeiro e a prevalência da infecção/infestação parasitária, com prévia transformação angular dos dados de prevalência (ZAR, 1996).

As amostras dos hospedeiros foram separadas em seis intervalos de classe com amplitude de $3,5 \mathrm{~cm}$. A terminologia ecológica usada é a recomendada por Bush et al. (1997). Todos os valores que correspondem à média de alguma variável são acompanhados do respectivo desvio padrão. 0 nível de significância estatística adotado foi $P<0,05$.

\section{RESULTADOS}

Os espécimes de $E$. saurus mediram $38,1 \pm 5,1(28-49) \mathrm{cm}$ de comprimento total. Trinta e cinco ( $70 \%)$ espécimes de $E$. saurus estavam parasitados por, pelo menos, uma espécie de metazoário. Elops saurus é um novo registro de hospedeiro para a larva do nematoide Contracaecum sp..

Um total de 159 espécimes de parasitos pertencentes a três espécies foi coletado, com abundância média de 3,2 \pm 4,2 e com a riqueza parasitária de $0,90 \pm 0,73$ (1 - 3). 0 copépode Lernanthropus rathbuni foi o mais prevalente e abundante (Tabela 1). Os copépodes e o nematoide corresponderam a $95,6 \%$ e $4,4 \%$ do total de espécimes de parasitos coletados, respectivamente. 
Os componentes da comunidade parasitária do E. saurus apresentaram o típico padrão de distribuição superdisperso (Tabela 2). Lernanthropus rathbuni apresentou a maior frequência de dominância e o maior valor de dominância relativa média (Tabela 3). Não foi observada correlação entre o comprimento total do hospedeiro e a prevalência e abundância parasitárias (Tabela 4). A abundância média $\left(r_{s}=0,184 ; P=0,199\right)$ e a riqueza parasitária $\left(r_{s}=0,204 ; P=0,153\right)$ não apresentaram relação com o comprimento total do hospedeiro.

Tabela 1 - Prevalência, amplitude da intensidade, intensidade média, abundância média e local de infecção/infestação dos metazoários parasitos de Elops saurus.

\begin{tabular}{lcccc}
\hline Parasitos & $\begin{array}{c}\text { Prevalência } \\
(\%)\end{array}$ & $\begin{array}{c}\text { Amplitude da } \\
\text { intensidade }\end{array}$ & Intensidade média & Abundância média \\
\hline Nematoda & & & & \\
\hline Contracaecum sp. (Larva) & 12 & $1-2$ & $1,1 \pm 0,4$ & $0,1 \pm 0,4$ \\
\hline Copepoda & & & & \\
\hline Caligus productus & 10 & $1-2$ & $1,2 \pm 0,4$ & $0,1 \pm 0,3$ \\
\hline Lernanthropus rathbuni & 68 & $1-19$ & $4,3 \pm 4,2$ & $2,9 \pm 4$ \\
\hline
\end{tabular}

Fonte: dos autores.

Tabela 2 - Índice de dispersão (ID) e do estatístico $d$ dos metazoários parasitos de Elops saurus.

\begin{tabular}{lcc}
\hline Parasitos & $\begin{array}{c}\text { Índice de dispersão } \\
\text { (ID) }\end{array}$ & D \\
\hline Contracaecum sp. (Larva) & 1,164 & 0,840 \\
\hline Caligus productus & 1,233 & 1,152 \\
\hline Lernanthropus rathbuni & 5,575 & $13,534 *$ \\
\hline Total & 5,628 & $13,631 *$ \\
\hline
\end{tabular}

(*) Valores significativos.

Fonte: dos autores.

Tabela 3 - Frequência de dominância, frequência de dominância compartilhada e dominância relativa média dos componentes das infracomunidades dos metazoários parasitos de Elops saurus.

\begin{tabular}{lccc}
\hline Parasitos & $\begin{array}{c}\text { Freqüência de } \\
\text { dominância }\end{array}$ & $\begin{array}{c}\text { Frequiência de dominância } \\
\text { compartilhada }\end{array}$ & $\begin{array}{c}\text { Dominância } \\
\text { relativa média }\end{array}$ \\
\hline Contracaecum sp. (Larva) & 0 & 1 & $0,03 \pm 011$ \\
\hline Caligus productus & 0 & 1 & $0,06 \pm 0,19$ \\
\hline Lernanthropus rathbuni & 32 & 2 & $0,91 \pm 0,22$ \\
\hline
\end{tabular}

Fonte: dos autores.

Tabela 4 - Valores do coeficiente de correlação por postos de Spearman $\left(r_{s}\right)$ e do coeficiente de correlação de Pearson ( $r$ ) para avaliar o relacionamento entre o comprimento total do Elops saurus e a abundância e a prevalência dos componentes de sua comunidade parasitária.

\begin{tabular}{lcccc}
\hline Parasitos & $\mathbf{r}_{\mathrm{s}}$ & $\mathbf{P}$ & $\mathbf{r}$ & $\mathbf{P}$ \\
\hline Contracaecum sp. (Larva) & 0,121 & 0,402 & 0,062 & 0,906 \\
\hline Caligus productus & 0,173 & 0,229 & 0,663 & 0,151 \\
\hline Lernanthropus rathbuni & 0,166 & 0,247 & $-0,278$ & 0,593 \\
\hline
\end{tabular}

(*) Valores significativos. $(P)$ nível de significância

Fonte: dos autores. 


\section{DISCUSSÃO}

Os resultados obtidos no presente estudo indicam que a comunidade de metazoários parasitos de $E$. saurus foi caracterizada pela presença de larvas de nematoide anisaquídeo, dominância do copépode $L$. rathbuni e baixa riqueza parasitária.

Os nematoides anisaquídeos, no presente estudo representados por larvas de Contracaecum sp., são componentes frequentes na maioria das comunidades de metazoários parasitos de peixes marinhos do sudeste brasileiro (TAVARES, LUQUE, 2006; LUQUE et al., 2011).

Pertencentes à família Anisakidae Skrjabin \& Karokhin, 1945 esses nematoides são parasitos de peixes marinhos e dulcícolas, mamíferos marinhos, répteis e aves piscívoras. Os anisaquídeos são dependentes do ambiente aquático para o seu desenvolvimento e transmissão através da cadeia alimentar. 0 ciclo biológico das espécies dessa família usualmente envolvem invertebrados aquáticos e peixes como hospedeiros intermediários e paratênicos (ANDERSON, 2000; TAVARES, LUQUE, 2006).

No Brasil, a ocorrência desses parasitos está associada aos gêneros Anisakis, Contracaeum, Pseudoterranova, Terranova, Hysterothylacium e Raphidascaris (TAVARES, LUQUE, 2006; LUQUE et al., 2011). A presença desse nematoide em Elops saurus é um indicativo de que ele está em uma posição intermediária da cadeia alimentar (LUQUE, POULIN, 2004). Além disso, o hábito alimentar desse peixe, composto de crustáceos e peixes (FIGUEIREDO, MENEZES, 1978; ADAMS et. al., 2013), reforça tal fato.

A comunidade parasitária de $E$. saurus apresentou a dominância do ectoparasito $L$. rathbuni. A maioria dos estudos sobre as comunidades de metazoários parasitos de peixes marinhos do sudeste brasileiro relatam o padrão de dominância dos endoparasitos, sejam esses estágios larvais ou adultos (MARQUES, ALVES, 2011; GONÇALVES, ALVES, 2012).

Entretanto, um padrão semelhante ao observado no presente estudo foi registrado por Tavares et al. (2001) com o peixe conhecido como olho-de-cão Priacanthus arenatus (Cuvier, 1829) (Osteichthyes: Priacanthidae) proveniente do litoral do Estado do Rio de Janeiro. Nesse caso, o copépode Hatschekia quadrabdominalis Yu, 1933 (Hatschekiidae) foi a espécie dominante.

No presente estudo, Lernanthropus rathbuni Wilson, 1922 (Siphonostomatoida: Lernanthropidae) foi a espécie dominante. Essa espécie de copépode, assim como Caligus productus Müller, 1785 (Siphonostomatoida: Caligidae), foi registrada parasitando E. saurus no Atlântico Norte (PEARSE, 1952).

Outra característica marcante na comunidade de metazoários parasitos de $E$. saurus foi a baixa riqueza. Apenas três espécies de parasitos foram registradas. Tal fato pode estar associado aos fatores abióticos da região onde os espécimes foram coletados e as características biológicas do hospedeiro (LUQUE et. al., 2004).

Entretanto, a escassez de estudos sobre a biologia de $E$. saurus no Brasil dificulta a realização de uma associação para justificar o padrão observado. Além disso, o resultado do presente estudo indica diferença na composição da fauna parasitária dos espécimes de $E$. saurus provenientes do Atlântico Sul e do Norte, onde, nesse último, foram registrados cestoides e digenéticos (LINTON, 1890; PALM, OVERSTREET, 2000; CORKUM, 1959; BULLARD, 2014). 


\section{AGRADECIMENTO}

Agradecemos ao Centro Universitário de Volta Redonda (UniFOA), pelo apoio financeiro.

\section{REFERÊNCIAS}

ADAMS, A. J.; HORODYSKY, A. Z.; MCBRIDE, R. S.; GUINDON, K.; SHENKER, J.; MACDONALD, T. C.; HARWELL, H. D.; WARD, R.; CARPENTER, K. Global conservation status and research needs for tarpons (Megalopidae), ladyfishes (Elopidae) and bonefishes (Albulidae). Fish and Fisheries, n. 8, v. 2, p. 1 - 32. 2013.

AMATO, J. F. R.; BOEGER, W. A.; AMATO, S. B. Protocolos para Laboratório - Coleta e Processamento de Parasitos de Pescado. Imprensa Universitária, Universidade Federal Rural do Rio de Janeiro, p. 81, 1991.

ANDERSON, R. C. Nematode Parasites of Vertebrates. Their Development and Transmission. 2. ed. CABI Publishing, Wallingford, Oxon (UK), p. 650, 2000.

BULLARD, S. A. Blood Flukes (Digenea: Aporocotylidae) of Elopomorphs: Emendation of Paracardicoloides, Supplemental Observations of Paracardicoloides yamagutii, and a New Genus and Species from Ladyfish, Elops saurus, (Elopiformes: Elopidae) in the Gulf of Mexico. Journal of Parasitology. v. 100, n. 3, p. $305-316,2014$.

BUSH, A. O.; AHO, J. M.; KENNEDY, C. R. Ecological versus phylogenetic determinants of helminth parasite community richness. Evolutionary Ecology, v. 4, n. 1. p. 1-20, 1990.

BUSH, A. O.; LAFFERTY, K. D.; LOTZ, J. M.; SHOSTAK, A. W. Parasitology meets ecology on its own terms: Margolis et al. revisited. Journal of Parasitology, v. 83, n. 4, p. 575- 583, 1997.

CORKUM, K. C. Some trematode parasites of fishes from the Mississippi gulf coast. Proceedings of the Louisiana Academy of Science 22:17-29, 1959.

EIRAS, J. C., TAKEMOTO, R. M., PAVANELLI, G. C. Métodos de estudo e técnicas laboratoriais em parasitologia de peixes. Maringá: UEM. p. 171, 2000.

FIGUEIREDO, J. L.; MENEZES, N. A. Manual de peixes marinhos do sudeste do Brasil. II. Teleostei (1). São Paulo, Museu de Zoologia da Universidade de São Paulo. p. 110, 1978.

GONÇALVES, P. H. S.; ALVES, D. R. Ecologia da comunidade de metazoários parasitos do xixarro, Trachurus lathami Nichols, 1920 (Osteichthyes: Carangidae) do litoral do Estado do Rio de Janeiro, Brasil. Cadernos UniFOA, 20: 105-113, 2012.

LINTON, E . Notes on entozoa of marine fishes of New England. Part 2. Cestodes. Ann. Rep. U.S. Fish Comm, 15:719-899, 1890.

LOPES, P. R. D. Catálogo dos Peixes Marinhos do Laboratório de Ictiologia da Universidade Federal do Rio de Janeiro. Parte I: Chondrichthyes (Rajiformes). Teleostei (Elopiformes a Dactylopteriformes). Revista Brasileira de Zoologia, v. 6, n.2, p. 201-217, 1989. 
LUDWIG, J. A.; REYNOLDS, J. F. Statistical Ecology: a primer on methods and computing. New York: Wiley-Interscience Publications, p. 337, 1988.

LUQUE, J. L.; POULIN, R. Use of fish as intermediate hosts by helminth parasites: A comparative analysis. Acta Parasitologica, 49 (4): 353-361, 2004.

LUQUE, J. L.; AGUIAR, J. C.; VIEIRA, F. M.; GIBSON, D. I.; SANTOS, C. P. Checklist of Nematoda associated with the fishes of Brazil. Zootaxa, 3082: 1-88, 2011.

LUQUE, J. L.; MOUILLOT, D.; POULIN, R. Parasite biodiversity and its determinants in coastal marine teleost fishes of Brazil. Parasitology, 128: 671-682, 2004.

MARQUES, L. C.; ALVES, D. R. Ecologia da comunidade de metazoários parasitos do dourado, Coryphaena hippurus Linnaeus, 1758, (Osteichthyes: Coryphaenidae) do litoral do Estado do Rio de Janeiro, Brasil. Cardernos UniFOA, 16: 111-122, 2011.

McBRIDE; R. S.; ROCHA; C. R.; RUIZ-CARUS, R.; BOWEN, B. W. A new species of ladyfish, of the genus Elops (Elopiformes: Elopidae), from the western Atlantic Ocean. Zootaxa, 2346, 29-41. 2010.

MENEZES, N. A.; BUCKUP, P. A.; FIGUEIREDO, J. L.; MOURA, R. L. Catálogo das Espécies de Peixes Marinhos do Brasil. São Paulo: Museu de Zoologia USP, p. 31, 2003.

NELSON, J. S. Fishes of the World. 4. ed. John Wiley \& Sons, New York, p. 601, 2006.

PALM, H. W.; OVERSTREET, R. M. New records of trypanorhynch cestodes from the Gulf of Mexico, including Kotorella pronosoma (Stossich, 1901) and Heteronybelinia palliata (Linton, 1924) comb. $\mathrm{n}$. Folia Parasitologica 47: 293-302, 2000.

PEARSE, A. S. Parasitic Crustacea from the Texas coast. Publs. Inst. Mar. Sci. Univ. Tex., 2: 5 - 42, 1952.

ROHDE, K.; HAYWARD, C.; HEAP, M. Aspects of the ecology of metazoan ectoparasites of marine fishes. International Journal for Parasitology, v. 25, n. 8, p. 945-970, 1995.

TAVARES, L. E. R.; LUQUE, J. L. Sistemática, biologia e importância em saúde coletiva de larvas de Anisakidae (Nematoda: Ascaridoidea) parasitas de peixes ósseos marinhos do Estado do Rio de Janeiro, Brasil. In: A. T. SILVA-SOUZA (Ed.), Sanidade de Organismos Aquáticos no Brasil. Maringá: Abrapoa, p. 297-328, 2006.

TAVARES, L. E. R.; LUQUE, J. L.; NETO, S. L. B. Ecologia da comunidade de metazoários parasitos do olho-de-cão Priacanthus arenatus (Cuvier, 1829) (Osteichthyes: Priacanthidae) do litoral do Estado do Rio de Janeiro, Brasil. Revista Brasileira de Zoociências, 3: 45-59, 2001.

WHITEHEAD, P. J. P., VERGARA, R. R. Elopidae. In W. Fischer (ed.) FAO species identification sheets for fishery purposes. Western Central Atlantic (Fishing Area 31). Vol. 2, 1978.

ZAR, J. H. Biostatistical Analysis. Upper Saddle River: Prentice-Hall, p. 662, 1996. 